\title{
Some New 2-Designs from a Wreath Product on 18 Points*
}

\author{
SneŽAna MatiĆ-KeKiĆ And Vojislav Mudrinski
}

\begin{abstract}
The total of 22 2-designs on 18 points have been found. All these designs have the same group as an automorphism group. This group can be represented as the wreath product of $G$ and $H$, where $G$ denotes the subgroup of order 3 of $\operatorname{PSL}(2,2)$ and $H$ denotes the subgroup of order 12 of PSL $(2,5)$.

The $2-(18,7,42 \cdot s)$ designs for $s \in\{15,19,25,27,30,37,38,50\}$ and the $2-(18,8,28 \cdot s)$ designs for $s \in\{27,44,46,48,50,53,54,57,59,61,73,77$, $80,81\}$ have been detected. Up to our knowledge, 16 of these 22 found designs are new.
\end{abstract}

\section{INTRODUCTION}

A $t$ - $(v, k, \lambda)$ design is a collection $\mathbf{B}$ of $k$-subsets (called block) of a $v$-element set $\Delta$ of points, which satisfies the property that each $t$-element subset of $\Delta$ is in exactly $\lambda$ blocks. We also require that blocks are not repeated.

Given a group $M$ acting on $\Delta$, the Kramer-Mesner method [7] searches for $t-(v, k, \lambda)$ designs having $M$ as an automorphism group. The group $M$ is a subgroup of the full automorphism group and the collection $\mathbf{B}$ is a union of $M$-orbits of $k$-subsets (shortly: $k$ - $M$-orbits).

The method includes a construction of $t-M$-orbits and $k$ - $M$-orbits, computation of the orbit incidence matrix $\Lambda(t, k)=\lambda_{i, j}$ (where $\lambda_{i, j}$ denotes the number of blocks from the $j$-th $k$ - $M$-orbit, containing a specified set from the $i$-th $t$ - $M$-orbit), and design recognition (by finding those proper sets of the column-set of $\Lambda(t, k)$, that have the uniform row sum $\lambda$ ).

In this paper we are going to apply the Kramer-Mesner method to the wreath product of some groups. This product will be described and discussed in the following section.

2000 Mathematics Subject Classification. Primary: 05B05; Secondary: 65D17.

Key words and phrases. Designs, Wreath product, Automorphism group.

* The work of the first author was supported by Serbian Government under Grant No. BTR20065.B and under Grant No. BTR20078.B. 


\section{Construction}

Let $G$ and $H$ be two groups acting on the ground-sets $\Gamma$ and $\Omega$ respectively. The wreath product $G \succ H$ is the group which acts on $\Gamma \times \Omega$ as follows ([6], Ch. I, Th. 15.3.):

$$
(i, j)(\mathbf{f}, h)=\left(i^{\mathbf{f}(j)}, j^{h}\right),
$$

where $h \in H, \mathbf{f}$ is a mapping from $\Omega$ into $G,(\mathbf{f}, h) \in G \imath H, i \in \Gamma, j \in \Omega$.

Groups $G$ and $H$ will be defined as some transitive subgroups of $P S L(2,2)$ and $P S L(2,5)$, respectively. The group $P S L(2,2)$ acts 2-transitively on the projective plane $\Gamma$ of order 2 and is isomorphic to the group $G L(2,2)$ of all regular $2 \times 2$ matrices over $G F(2)$. Similarly, the group $P S L(2,5)$ acts on the projective plane $\Omega$ of order 6 and is isomorphic to the groups $G L(2,5)$ of all regular $2 \times 2$ matrices over $G F(5)$.

The group $P S L(2,2)$ is also isomorphic to the symmetric group $S_{3}$. We choose $G$ to be its alternating subgroup $A_{3}$, which is known to act transitively on $\Gamma$. We choose $H$ to be the normalizer of a Klein subgroup of $\operatorname{PSL}(2,5)$. This normalizer is known ([6], Ch. II, Lemma 8.16) to be of order 12 .

The group $P S L(2,2) \curlywedge P S L(2,5)$ of order $6^{6} \cdot 60$ is not computationally tractable. Combining the facts that

- the Kramer-Mesner method searches (for) designs as some unions of orbits;

- orbits by action of a group are partitioned into orbits by action of its subgroups ([2], Lemma 1),

it follows that no design arising by action of $P S L(2,2) \curlywedge P S L(2,5)$ will be missed by considering the action of $G$ ? $H$.

Since we found that the wreath product is reach in designs [4], [3],[1] we were motivated to continue this investigation.

\section{Designs With Wreath PROduCt on 18 Points}

Throughout the remaining part of this paper, considerations will be restricted to the automorphism group $G$ l $H$. Therefore, the notation " $k-(G)$ $H$ )-orbit" will be abbreviated to " $k$-orbit".

Design recognition, i.e. search over the matrices $\Lambda(2, k)$, has been very facilitated by the fact that the matrices have many repeated columns. We use these repetitions to abbreviate notations for $\Lambda(2, k)$ by writing only the non-repeating columns, with the additional fourth row containing data on multiplicity (on the number of repetitions). The abbreviated tables will be denoted by $T(k)$; the first and the fourth row in such a table will be separated by a horizontal line. The ordinal numbers of columns of matrix $\Lambda(2, k)$ are listed in the first row on the $T(k)$.

We have performed the complete search for 2-designs with the automorphism group $G$ ? $H$. 
3.1. Matrices $\Lambda(2, k)$. Since we found only $2-(18,7, \lambda)$ and $2-(18,8, \lambda)$ designs with the automorphism group equals to the wreath product $G$ ? $H$, we list only the matrices $\Lambda(2, k) k=7,8$. It turns out that there exist 3 2 -orbits, 447 -orbits and 528 -orbits. We list the matrices $\Lambda(2, k) k=7,8$ in their abbreviated forms $T(k)$ (frequencies of the columns are listed in the last row of the tables):

TABLE 1. $T(7) \lambda_{\max } / 2=2184$.

\begin{tabular}{|c|c|c|c|c|c|c|c|c|c|c|c|c|c|c|c|c|c|c|c|}
\hline 1. & 2. & 3. & 4. & 5. & 6. & 7. & 8. & 9. & 10. & 11. & 12. & 13. & 14. & 15. & 16. & 17. & 18. & 19. & 20 \\
\hline 1 & 4 & 10 & 6 & 15 & 36 & 54 & 5 & 16 & 33 & 42 & 45 & 48 & 144 & 126 & 189 & 405 & 72 & 432 & 648 \\
6 & 4 & 24 & 8 & 42 & 60 & 108 & 0 & 0 & 72 & 36 & 24 & 12 & 72 & 144 & 270 & 432 & 36 & 324 & 648 \\
6 & 12 & 30 & 15 & 36 & 72 & 81 & 12 & 30 & 72 & 72 & 72 & 72 & 162 & 162 & 162 & 324 & 81 & 32 & 243 \\
\hline 2 & 2 & 2 & 2 & 2 & 2 & 2 & 2 & 2 & 2 & 4 & 4 & 2 & 2 & 4 & 2 & 2 & 1 & 2 & 1 \\
\hline
\end{tabular}

TABLE 2. $T(8) \lambda_{\max } / 2=2285$.

\begin{tabular}{|cccccccccccc|}
\hline 1. & 2. & 3. & 4. & 5. & 6. & 7. & 8. & 9. & 10. & 11. & 12 \\
\hline 1701 & 405 & 270 & 567 & 189 & 180 & 513 & 171 & 153 & 63 & 60 & 21 \\
1620 & 486 & 270 & 432 & 108 & 144 & 648 & 180 & 252 & 24 & 36 & 4 \\
972 & 243 & 243 & 486 & 216 & 216 & 486 & 216 & 216 & 90 & 90 & 36 \\
\hline 1 & 1 & 3 & 2 & 2 & 3 & 2 & 4 & 2 & 4 & 2 & 1 \\
\hline
\end{tabular}

\begin{tabular}{|cccccccccccc|}
\hline 13. & 14. & 15. & 16. & 17. & 18. & 19. & 20. & 21. & 22. & 23. & 24. \\
\hline 57 & 51 & 19 & 13 & 7 & 36 & 24 & 45 & 16 & 3 & 5 & 2 \\
48 & 72 & 12 & 36 & 0 & 72 & 42 & 96 & 24 & 10 & 8 & 6 \\
90 & 90 & 36 & 36 & 14 & 54 & 45 & 90 & 36 & 9 & 14 & 7 \\
\hline 2 & 4 & 4 & 1 & 2 & 1 & 2 & 2 & 1 & 2 & 2 & 2 \\
\hline
\end{tabular}

3.2. $\mathbf{2}-(\mathbf{1 8}, \mathbf{7}, \lambda)$ designs. We searched over the matrix $T(7)$ without using multiplicity. In this way we obtained parameters $\lambda \in\{630,798,1050,1554\}$. We additionally searched over the matrix $T(7)$ using multiplicity. In this manner we found $\lambda$ parameters 1134, 1360,1596, 2100.

In the following table we present choice of the index of columns (without using multiplicity) of the matrix $T(7)$ for designs $2-(18,7,42 \cdot s)$ $s \in\{15,19,25,37\}:$

TABLE 3 .

\begin{tabular}{|c|c|}
\hline$\lambda=630=42 \cdot 15$ & 1.2 .4 .7 .9 .14 .17$. \\
\hline$\lambda=798=42 \cdot 19$ & 2.3 .4 .7 .9 .11 .12 .16 .19$. \\
\hline$\lambda=1050=42 \cdot 25$ & 1.2 .3 .4 .6 .7 .8 .9 .10 .13 .17 .19$. \\
\hline$\lambda=1554=42 \cdot 37$ & 3.6 .7 .8 .10 .13 .14 .15 .16 .17 .18 .19$. \\
\hline
\end{tabular}


Since the supposed columns for parameters $\lambda=630,798,1050$ have multiplicity greater than 1 , we found the designs with parameters $\lambda=1360,1596,2100$, using multiplicity 2 .

In the following table we give the choice of columns of designs $2-(18,7,42 \cdot 27)$ together with its multiplicity.

TABLE 4.

\begin{tabular}{|llll|}
\hline \multicolumn{5}{|c|}{$\lambda=1134=42 \cdot 27$} \\
\hline $14 . \cdot 2$ & $15 . \cdot 2$ & $16 . \cdot 1$ & $17 . \cdot 1$ \\
\hline
\end{tabular}

3.3. $\mathbf{2 - ( 1 8 , 8 , \lambda )}$ designs. We searched over the matrix $T(8)$ without using multiplicity. In this way we obtained parameters $\lambda \in\{28 \cdot s$, $s=27,44,46,48,50,53,57,59,61,73,77,80,81\}$. In [5] the results for $\lambda$ are found: $\lambda \in\{28 \cdot s, s \bmod 2=0\}$. In the following table the choice of index of columns for new $\lambda$ parameters is given:

TABLE 5.

\begin{tabular}{|c|c|}
\hline$\lambda=756=28 \cdot 27$ & 4.9 .18$. \\
\hline$\lambda=1484=28 \cdot 53$ & 2.4 .5 .9 .10 .15 .16 .17 .20 .21 .23 .24$. \\
\hline$\lambda=1596=28 \cdot 57$ & 2.4 .5 .6 .9 .12 .16 .20 .21 .23 .24$. \\
\hline$\lambda=1652=28 \cdot 59$ & 3.4 .5 .7 .15 .16 .18 .19 .21 .23$. \\
\hline$\lambda=1708=28 \cdot 61$ & 2.3 .4 .9 .10 .11 .12 .13 .14 .15 .19 .21 .24$. \\
\hline$\lambda=2044=28 \cdot 73$ & 2.3 .4 .5 .6 .8 .9 .12 .16 .17 .18 .19 .22 .23$. \\
\hline$\lambda=2156=28 \cdot 77$ & 2.4. 5. 6. 7. 10.11.12.14.17. 18.20.21. 22. \\
$\lambda=2268=28 \cdot 81$ & 2. 4. 5.6.7.8.10.11.14.19.20. \\
\hline
\end{tabular}

Theorem. Let $G$ be a subgroup of order 3 of PSL $(2,2)$ and let $H$ be the normalizer of a Klein subgroup of $\operatorname{PSL}(2,5)$. Then there exist $2-(18,7, \lambda)$ designs for

$$
\lambda \in\{42 \cdot s, s=15,19,25,27,30,37,38,50\}
$$

and the $2-(18,8, \lambda)$ designs for

$$
\lambda \in\{28 \cdot s, s=27,44,46,48,50,53,54,57,59,61,73,77,80,81\}
$$

with automorphism group which is equal to the wreath product $G$ ? $H$.

The direct action of this wreath product on the Cartesian product of the projective line of order 2 and the projective plane of order 6 does not give 2- $(18, k, \lambda)$ designs with other values of $\lambda$. 


\section{REFERENCES}

[1] D.M. Acketa, V. Mudrinski, Two 5-designs on 32 points, Discrete Math., 163 (1997), 209-210.

[2] D.M. Acketa V. Mudrinski, A family of 4-designs on 26 points, Comment. Math. Univ. Carolinae, Vol. 37, No. 4 (1996), 843-860.

[3] D.M. Acketa, V. Mudrinski, A family of 4-designs on 38 points, Ars Combinatoria, 53 (1999), 93-110.

[4] D.M. Acketa, V. Mudrinski, S. Matić-Kekić, A large collection of designs from a wreath product on 21 points, Ars Combinatoria, 54 (2000), 109-118.

[5] Y.M. Chee, C.J. Colbourn, D.L. Kreher, Simple t-designs with $v \leq 30$, Ars Combinatoria, 29 (1990), 193-258.

[6] B. Huppert, Endliche Gruppen I, Die Grundlehren der mathematischen Wissenchaften, Band 134, (1967), Springer-Verlag, Berlin, Heidelberg, New York, XII, 793pp.

[7] E.S. Kramer, D.M. Mesner, t-designs on hypergraphs, Discrete Math., 15 (1976), 263-296.

\author{
Sneżana Matić-Kekić \\ Lecturing Desk of Mathematics \\ Department of Agricultural Engineering \\ Faculty of Agriculture \\ UNIVERSITY OF NOVI SAD \\ Trg Dositeja Obradovića 8 \\ 21000 Novi SAD \\ SERBIA \\ E-mail address: snmk@polj.ns.ac.rs
}

